\title{
Letter to the Editor: 'Long-Term Results after Laparoscopic Sleeve Gastrectomy with Concomitant Posterior Cruroplasty: 5-Year Follow-up"
}

\author{
Thomas E. Baldock ${ }^{1}$ (D) $\cdot$ Leo R. Brown ${ }^{2} \cdot$ Ross C. McLean ${ }^{3}$ \\ Received: 14 December 2019 / Accepted: 13 February 2020 / Published online: 5 March 2020 \\ (C) 2020 The Society for Surgery of the Alimentary Tract
}

\section{Dear Authors,}

We read with interest your paper "Long-term results after laparoscopic sleeve gastrectomy with concomitant posterior cruroplasty: 5-year follow-up". ${ }^{1}$ We would appreciate some clarification of your results.

It is crucial to note that authors have directly compared two groups of patients whom have two different operations for two different sized hiatal hernias; comparing the proverbial "apples and oranges." It is therefore difficult to conclude if there is a place for mesh reinforcement in this group of patients. It would have been more meaningful if comparisons were with small defect suture vs. mesh or large defect suture vs. mesh as the pathology would be comparable. We are unable to draw conclusions on the benefits of mesh repair, which is particularly important, as the authors were using mesh in patients whom were initially asymptomatic. The use of mesh is not without its own complications, and the routine use of mesh is yet to be proven.

Another concern regarding their reporting is that the authors state 37/96 patients had a mild-to-moderate diagnosis of GERD preoperatively (Group A, 17/48; Group B, 20/48). While it is not stated in their table, the reader would infer the remainder of the cohort was asymptomatic from the statement "from all 37 initially symptomatic." At the end of the 5-year follow-up, 6 patients in Group A had GERD recurrence compared with 9 patients in Group B. The authors conclude that they observed a remission rate of $85 \%$ in Group A and $80 \%$ in

Thomas E. Baldock

mr.thomas.baldock@gmail.com

1 Department of General Surgery, Auckland Hospital, 2 Park Road, Grafton, Auckland 1023, New Zealand

2 Department of General Surgery, Dumfries \& Galloway Royal Infirmary, A75, Cargenbridge, Dumfries DG2 8RX, UK

3 Department of General Surgery, University Hospital of North Tees, Hardwick Road, Stockton-on-Tees TS19 8PE, UK
Group B at 5 years. However, these proportions incorrectly include the denominator of initially asymptomatic patients in their calculations. Excluding these patients, the recurrence rate at 5 years appears to be much higher for Group A of 35\% (6/17) and Group B of 45\% (9/20). Consequently, the remission rate is also much lower (Group A, 65\% and Group B, $55 \%)$. It is also important to note that 8 patients in Group A and 4 in Group B underwent revisional procedures for refractory GERD (two in each group were then lost to follow-up). This means that of the people who had GERD pre-operatively, and excluding the patients whom were lost to follow-up, 35\% were converted to bypass in Group A (6/17) and 10\% in Group B $(2 / 20)$. This is a significant proportion of patients, which in turn, raises the question on whether a sleeve gastrectomy was the "right" operation for them.

Yours sincerely,

The Authors

\section{Compliance with Ethical Standards}

Conflict of Interest The authors declare that they have no conflict of interest.

\section{References}

1. Boru CE, Coluzzi MG, de Angelis F, Silecchia G. Long-Term Results After Laparoscopic Sleeve Gastrectomy with Concomitant Posterior Cruroplasty: 5-Year Follow-up. J Gastrointest Surg [Internet]. 2019 Aug 13; Available from: https://doi.org/10.1007/ s11605-019-04355-1

2. Zhang C, Liu D, Li F, Watson DI, Gao X, Koetje JH, Luo T, Yan C, Du X, Wang Z. Systematic review and meta-analysis of laparoscopic mesh versus suture repair of hiatus hernia: objective and subjective outcomes. Surg Endosc. 2017 Dec 1;31(12):4913-22.

Publisher's Note Springer Nature remains neutral with regard to jurisdictional claims in published maps and institutional affiliations. 